\title{
A garden of orchids: a generalized Harper equation at quadratic irrational frequencies
}

\author{
B. D. Mestel \\ Department of Computing Science and Mathematics \\ University of Stirling \\ Stirling \\ FK9 4LA, UK \\ B.D.Mestel@maths.stir.ac.uk \\ A. H. Osbaldestin \\ Department of Mathematics \\ University of Portsmouth \\ Portsmouth \\ PO1 3HE, UK \\ andrew.osbaldestin@port.ac.uk
}

July 20, 2004

\begin{abstract}
We consider a generalized Harper equation at quadratic irrational flux, showing, in the strong coupling limit, the fluctuations of the exponentially decaying eigenfunctions are governed by the dynamics of a renormalization operator on a renormalization strange set. This work generalizes previous analyses which have considered only the golden mean case. Projections of the renormalization strange sets are illustrated analogous to the "orchid" present in the golden mean case.
\end{abstract}

\section{Introduction}

The generalized Harper equation [9]

$$
\begin{aligned}
(1+\alpha \cos (2 \pi(\omega(i+1 / 2)+\phi))) \psi_{i+1}+(1 & +\alpha \cos (2 \pi(\omega(i-1 / 2)+\phi))) \psi_{i-1} \\
& +2 \lambda \cos (2 \pi(i \omega+\phi)) \psi_{i}=E \psi_{i},
\end{aligned}
$$

models an electron in a two-dimensional lattice in a transverse magnetic field in the limits of strong (weak) potential and weak (resp. strong) field. The parameters $\omega, \phi$, and $\lambda$ represent, respectively, the magnetic flux per unit cell, the wave-number of the plane wave in the transverse direction, and the ratio of the length of the unit cell in the direction of the vector potential and its length in the transverse direction. The parameter $\alpha$ measures the next-nearest-neighbour interaction strength, in whose absence we have the standard Harper equation [10]. (For earlier studies of the spectrum of this model see [4], [11], [27].)

In the localized regime $(\lambda>1)$ of the standard Harper equation $(\alpha=0)$, in the case of golden mean flux $(\omega=(\sqrt{5}-1) / 2)$, Ketoja and Satija [15] observe that the exponentially decaying eigenfunctions possess universal self-similar fluctuations determined by the strong coupling limit $\lambda \rightarrow \infty$. Ketoja and Satija explain this phenomenon in terms of a universal fixed point of a renormalization operator derived from their decimation scheme [13], [14]. Their observations have been put a firm footing by explicitly constructing a 
fixed point of the appropriate renormalization operator [22], and the generalization to quadratic irrationals of the form $\omega=\left(\sqrt{a^{2}+4}-a\right) / 2, a \in \mathbb{N}$, has also been achieved [5].

For the generalized Harper equation (1.1) the localized phase splits into two regions displaying different phenomena. For $\lambda>1, \alpha<1$ the fixed point of the standard Harper equation governs the fluctuations. The region $\lambda \geq \alpha \geq 1$ concerns us in this article. It appears [15] that the fluctuations are now governed by a renormalization strange set, which Ketoja and Satija call the orchid (see Figure 1) which, again, arises in the strong coupling limit $\lambda \rightarrow \infty$. We have recently shown how this orchid may be understood in terms of a shift map on an appropriate symbol space [25]. In fact the details of our work in [25] reveal that the orchid is composed of three copies of a more basic object, which we call the fundamental set. Figure 1 shows projections of these renormalization strange sets. Note that the orchid shown here is a reflection of that shown in [15] due to a different sign convention.
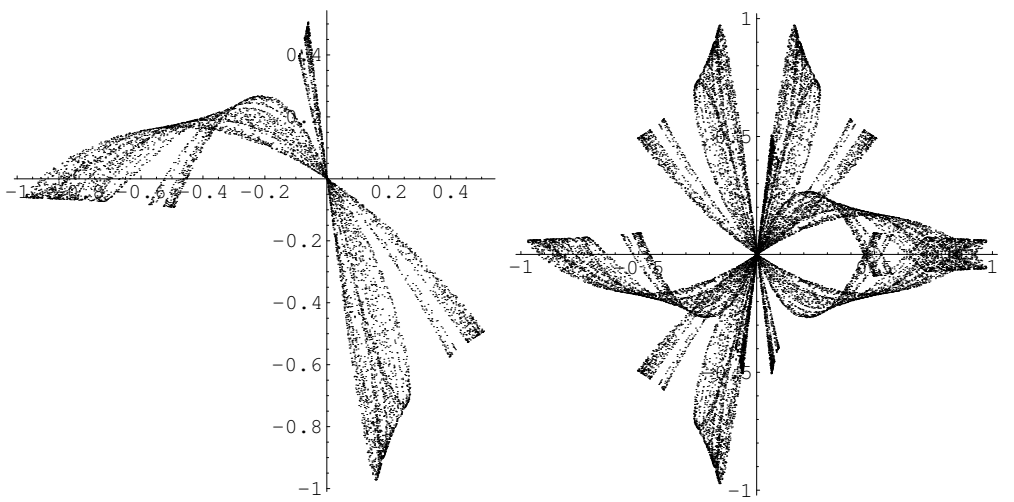

Figure 1: Projections of the golden mean fundamental set (left) and orchid (right)

The purpose of this article is to generalize our work in [25] to irrationals of the form $\omega=\left(\sqrt{a^{2}+4}-a\right) / 2$, $a \in \mathbb{N}$. The golden-mean case corresponds to $a=1$. The functional recurrence is

$$
t_{n}(x)=\prod_{i=0}^{a-1} t_{n-1}(-\omega x-i) t_{n-2}\left(\omega^{2} x+a \omega\right)
$$

with appropriate initial conditions. We may write (1.2) as a first-order recurrence by setting $u_{n}(x)=$ $t_{n-1}(-\omega x)$, so that we have the renormalization operator

$$
R:\left(\begin{array}{c}
u_{n-1}(x) \\
t_{n-1}(x)
\end{array}\right) \mapsto\left(\begin{array}{c}
t_{n-1}(-\omega x) \\
\prod_{i=0}^{a-1} t_{n-1}(-\omega x-i) u_{n-1}(-\omega x-a)
\end{array}\right) .
$$

We present renormalization strange sets akin to the Ketoja-Satija orchid for quadratic irrational frequencies other than the golden mean. Specifically, setting $\omega=\left(\sqrt{a^{2}+4}-a\right) / 2, a \in \mathbb{N}$, and iterating the recurrence (1.2) for appropriate initial conditions, we find convergence to strange invariant sets. Scaled projections of these sets to the plane are illustrated in Figures 2 and 3 below.

In [25] we presented a highly detailed, rigorous analysis of the structure of the Ketoja-Satija orchid in the golden-mean case. We showed that its mathematical structure is given essentially by the shift-map acting on a space of biinfinite sequences of symbols 0 and 1 (factored by a partnering relation corresponding to the symmetries of the cosine function in the generalized Harper equation (1.1)), together with dynamics on sign-pairs $( \pm 1, \pm 1)$. Our aim in this paper is to present a similar structure analysis for the case of general $a \in \mathbb{N}$. At present our analysis is still conjectural, but it likely that the methods used in [25] may be adapted to give rigorous backing to our results.

\section{Derivation of the renormalization equations}

We now recall from [5] the derivation of the renormalization functional recurrence, based on the so-called decimation method of Ketoja and Satija [13, 14, 15]. 


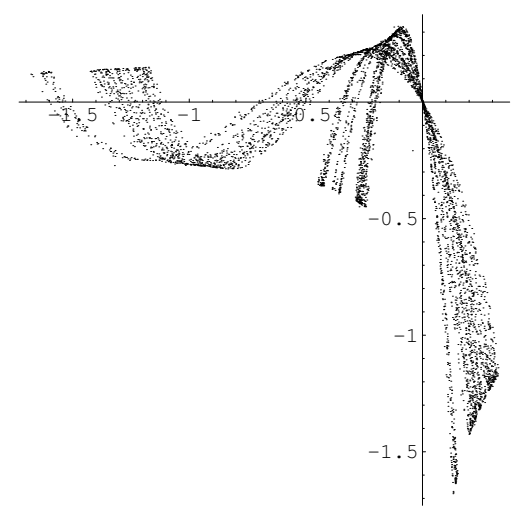

Figure 2: Projection of the fundamental set/orchid in the case $a=2$.

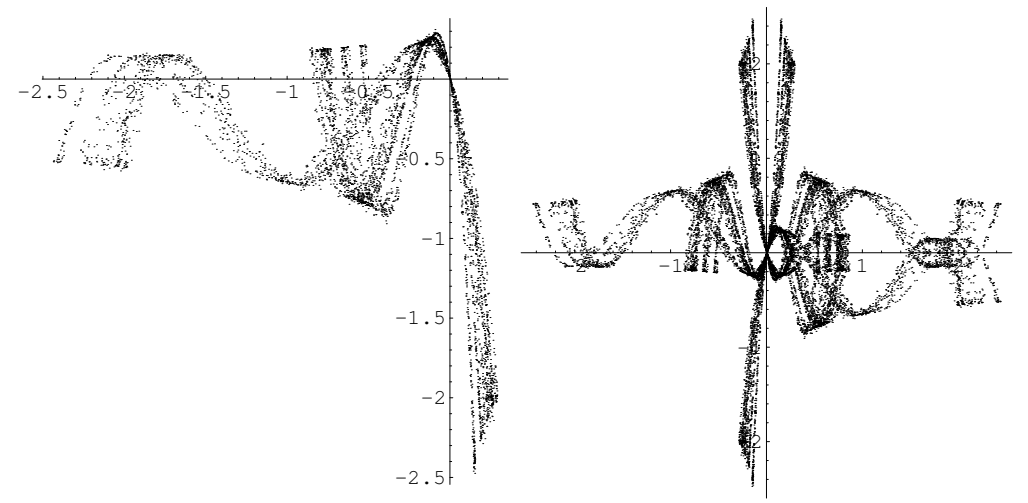

Figure 3: Projections of the fundamental set (left) and orchid (right) in the case $a=3$.

Let $a \in \mathbb{N}$ be fixed and let $\omega \in(0,1)$ have continued fraction expansion $[0 ; a, a, \ldots]$. Then $\omega=(-a+$ $\left.\sqrt{a^{2}+4}\right) / 2$, the positive root of the quadratic equation

$$
\omega^{2}+a \omega=1 .
$$

The rational convergents $p_{n} / q_{n}$ satisfy $p_{n}=q_{n-1}=G_{n}$ where $G_{n+1}=a G_{n}+G_{n-1}, G_{0}=0, G_{1}=1$. An important relation is the following:

$$
G_{n} \omega-G_{n-1}=-(-\omega)^{n}
$$

Let us consider the portion of the localized phase, $\lambda \geq 1, \lambda \geq \alpha$, for which $\alpha \geq 1$, and for which, by the result of Han et al [9], the exponential decay of the eigenfunction is given by the characteristic exponent

$$
\gamma=\log \left(\frac{\lambda}{\alpha}+\sqrt{\left(\frac{\lambda}{\alpha}\right)^{2}-1}\right) .
$$

We write

$$
\psi_{i}=e^{-\gamma|i|} \eta_{i},
$$

so that the generalized Harper equation (1.1) becomes, for $i>0$,

$$
\begin{aligned}
e^{-2 \gamma}(1+\alpha \cos (2 \pi(\omega(i+1 / 2)+\phi))) \eta_{i+1}+(1 & +\alpha \cos (2 \pi(\omega(i-1 / 2)+\phi))) \eta_{i-1} \\
& +2 e^{-\gamma} \lambda \cos (2 \pi(i \omega+\phi)) \eta_{i}=e^{-\gamma} E \eta_{i} .
\end{aligned}
$$

The quantity $\eta_{i}$ is the fluctuation of the wave function $\psi_{i}$ from the exponential decay law $\psi_{i} \approx e^{-\gamma|i|}$.

We now consider (2.5) in the strong-coupling limit $\lambda \rightarrow \infty$, at the so-called band edge $E=2 \lambda$. We note that $\lambda e^{-\gamma} \rightarrow \alpha / 2$. Setting $E=2 \lambda$ and taking the limit $\lambda \rightarrow \infty$ gives the recurrence

$$
\eta_{i-1}+\frac{\alpha(\cos (2 \pi(i \omega+\phi))-1)}{1+\alpha \cos (2 \pi(\omega(i-1 / 2)+\phi)))} \eta_{i}=0 .
$$


Following the method of Ketoja and Satija [15], we may define a so-called decimation by the relation:

$$
\eta_{i+G_{n}}=\hat{t}_{n}(i) \eta_{i}
$$

Note that this decimation differs slightly from the one in [15], as we have set the coefficient of $\eta_{i+G_{n+1}}$ to zero and we have changed the sign of $\hat{t}_{n}$. Consequently, equation (2.7) is only valid in the strong-coupling limit $\lambda \rightarrow \infty$.

A recurrence for $t_{n}$ may be obtained in the following way. We evaulate (2.7) with $i$ set equal to $i, i+G_{n}$, $i+2 G_{n}, \ldots, i+(a-1) G_{n}$, which gives the following set of equations:

$$
\begin{aligned}
\eta_{i+G_{n}} & =\hat{t}_{n}(i) \eta_{i} \\
\eta_{i+2 G_{n}} & =\hat{t}_{n}\left(i+G_{n}\right) \eta_{i+G_{n}} \\
\ldots \ldots & \ldots \ldots \ldots \\
\eta_{i+a G_{n}} & =\hat{t}_{n}\left(i+(a-1) G_{n}\right) \eta_{i+(a-1) G_{n}} .
\end{aligned}
$$

We now evaluate $(2.7)$ at $n-1$ with $i$ set equal to $i+a G_{n}$ giving

$$
\eta_{i+G_{n-1}+a G_{n}}=\hat{t}_{n-1}\left(i+a G_{n}\right) \eta_{i+a G_{n}} .
$$

Eliminating $\eta_{i+G_{n}}, \ldots, \eta_{i+a G_{n}}$ between these equations, we make use of the recurrence for the $G_{n}$, to obtain $\eta_{i+G_{n+1}}=\hat{t}_{n+1}(i) \eta_{i}$, where

$$
\hat{t}_{n+1}(i)=\left(\prod_{j=0}^{a-1} \hat{t}_{n}\left(i+j G_{n}\right)\right) \hat{t}_{n-1}\left(i+a G_{n}\right) .
$$

Setting the phase $\phi=0$, and evaluating (2.7) at $n=0$ and $n=1$, we obtain $\eta_{i}=\hat{t}_{0}(i) \eta_{i}, \eta_{i+1}=\hat{t}_{1}(i) \eta_{i}$, which, on comparing with $(2.6)$ at $i+1$, with $\phi=0$, gives

$$
\hat{t}_{0}(i)=1, \quad \hat{t}_{1}(i)=\frac{1+\alpha \cos (2 \pi(\omega(i+1 / 2)))}{\alpha(1-\cos (2 \pi(i+1) \omega))} .
$$

Following [15] we now transform from the discrete variable $i$ to a continuous variable $x$, by writing $x=$ $(-\omega)^{-n}\{i \omega\}$ where $\{\cdot\}$ denotes the fractional part. The transformation must be done with care since the definition of $x$ depends on the index $n$ of the function. We now write $t_{n}(x)=\hat{t}_{n}(i)$, where $t_{n}$ is a periodic function of period $\omega^{-n}$. Then, as in [5], we have, for $n>1$,

$$
\begin{aligned}
t_{n+1}(x) & =t_{n+1}\left((-\omega)^{-(n+1)}\{i \omega\}\right)=\hat{t}_{n+1}(i) \\
& =\left(\prod_{j=0}^{a-1} \hat{t}_{n}\left(i+j G_{n}\right)\right) \hat{t}_{n-1}\left(i+a G_{n}\right) \\
& =\left(\prod_{j=0}^{a-1} t_{n}\left((-\omega)^{-n}\left\{\left(i+j G_{n}\right) \omega\right\}\right)\right) t_{n-1}\left((-\omega)^{-(n-1)}\left\{\left(i+a G_{n}\right) \omega\right\}\right) \\
& =\left(\prod_{j=0}^{a-1} t_{n}\left(\left(-\omega(-\omega)^{-(n+1)}\left\{i \omega+j\left(-(-\omega)^{n}\right)\right\}\right)\right) t_{n-1}\left(\omega^{2}(-\omega)^{-(n+1)}\left\{\left(i \omega+a\left(-(-\omega)^{n}\right)\right)\right\}\right)\right. \\
& =\left(\prod_{j=0}^{a-1} t_{n}(-\omega x-j)\right) t_{n-1}\left(\omega^{2} x+a \omega\right)
\end{aligned}
$$

as required. (In deriving this equation we have implicitly used the periodicity of the function $t_{n}$ and (2.2).) The initial conditions for this recurrence are similar to those in [25] instead of those in [5]. In fact, using the definition of the variable $x$ and the periodicity of the cosine function, we have

$$
t_{0}(x)=1, \quad t_{1}(x)=\frac{1+\alpha \cos (2 \pi(-\omega x+\omega / 2))}{\alpha(1-\cos (2 \pi(-\omega x+\omega)))} .
$$

Careful numerical iteration of the recurrence (1.2) with the initial condition (2.19) leads to convergence to a renormalization strange set. 


\section{Renormalization analysis}

In this section we describe the mathematical structure that underlies the renormalization strange sets described above. Our first step is to define the iterated function system that is important to the analysis of the renormalization operator (1.3).

\subsection{The iterated function system and the fundamental interval}

We introduce the following notation. For $i=0, \ldots, a$, let

$$
\theta_{i}(x)=-\omega x-i
$$

Then we may rewrite the operator (1.3) as

$$
R:\left(\begin{array}{c}
u(x) \\
t(x)
\end{array}\right) \mapsto\left(\begin{array}{c}
t\left(\theta_{0}(x)\right) \\
\prod_{i=0}^{a-1} t\left(\theta_{i}(x)\right) u\left(\theta_{a}(x)\right)
\end{array}\right) .
$$

The functions $\left\{\theta_{0}, \theta_{1}, \theta_{2}, \ldots, \theta_{a}\right\}$ form an Iterated Function System (IFS), whose fixed-point set is the interval $I=[-\omega-a, 1]$, and which we refer to as the fundamental interval. The interval $I$ splits into subintervals $I_{i}$ given by $I_{0}=[-\omega, 1]$ and $I_{i}=[-\omega-i,-\omega-i+1]$ for $i=1, \ldots a$. Hence $\theta_{0}(I)=I_{0}$ and also we have $I_{i} \subseteq \theta_{i}(I)$ for $i=1, \ldots, a$. We can therefore define an inverse map $G: I \rightarrow I$ by

$$
G(x)=\theta_{i}^{-1}(x)=-\omega^{-1} x-i \omega^{-1}, \text { for } x \in I_{i} .
$$

Then $G\left(I_{i}\right)=I_{0} \cup \cdots \cup I_{a-1}$ for $i=1, \ldots, a$ and $G\left(I_{0}\right)=I$. See Figure 4 which shows $G$ for the case $a=2$. The functions $u$ and $t$ are defined on $I_{a}=[-\omega-a,-\omega-a+1]$ and $I_{0} \cup \cdots \cup I_{a-1}=[-\omega-a+1,1]$ respectively.

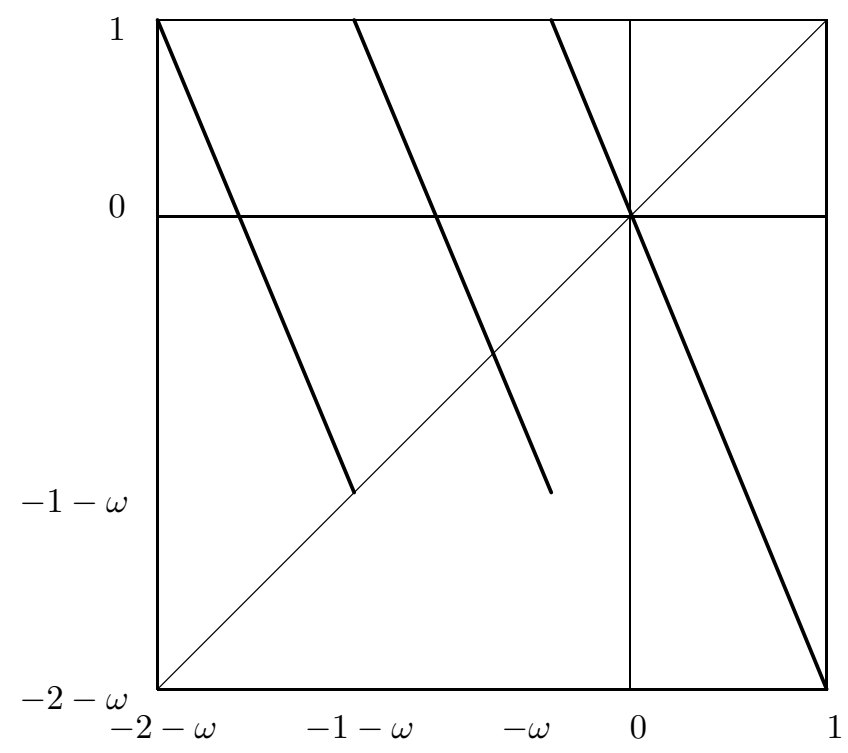

Figure 4: The function $G$ in the case $a=2$.

The significance of the function $G$ is that it governs the dynamics of the zeros of the functions $u$ and $t$ on their respective domains on the fundamental interval $I$. In turn the full dynamics of the functions $u$ and $t$ is essentially determined by their zeros on $I$.

\subsection{Shift spaces and partners}

A key goal of dynamical systems theory is to find well-understood models of chaotic systems that elucidate their structure. Indeed, the archetypal model of such a chaotic system is a Bernoulli shift on a space of symbol 
codes. The structure of the map $G$ as illustrated in Figure 4 suggests that a sub-shift of the Bernoulli shiftspace of symbols $0, \ldots, a$ is a suitable model for the orchid. This is indeed the case, although, as we shall see below, one must also take into account the symmetry of the cosine function in the initial condition (2.19) and the dynamics of the signs of the functions $u$ and $t$.

Let us first of all introduce the codes which form the basis of our construction. For fixed $a \in \mathbb{N}$ we define the code space

$$
\Sigma=\left\{\mathbf{c}=\left(c_{i}\right)_{i \in \mathbb{Z}}: c_{i} \in\{0,1, \ldots, a\}, c_{i}=a \Longrightarrow c_{i-1}=0\right\}
$$

This a space of biinfinite codes of symbols $\{0, \ldots, a\}$ with the single restriction that the symbol $a$ must be preceded by the symbol 0 . The reason for this restriction can be seen easily from the map $G$. The symbols $0, \ldots, a$ correspond to the subintervals $I_{0}, \ldots, I_{a}$ of the fundamental interval $I$, and the map $G$ determines the possible transitions between symbols. Indeed, the symbol $i$ is permitted to be followed by $j$ if, and only if, $I_{j} \subseteq G\left(I_{i}\right)$. Since the image under $G$ of each subinterval $I_{i}$ contains $I_{j}$ for all $j=0, \ldots a-1$, there are no restrictions needed in this case. However, since only $G\left(I_{0}\right)$ covers $I_{a}$, the symbol $a$ must be preceded by the symbol 0 as claimed. In [25], the codes of the symbols 0 and 1 were restricted so that no two consecutive symbols 1 were permitted. We note that in the case $a=1$ our condition is equivalent to that in [25]. Let us denote by $\sigma$ the left-shift map, defined for a code $\mathbf{c}=\left(c_{k}\right)_{k \in \mathbb{Z}}$ by $\sigma(\mathbf{c})_{k}=c_{k+1}$. Then, equipping $\Sigma$ with the standard metric, we have that $\sigma$ is a homeomorphism of $\Sigma$.

We now introduce a map $e_{n}: \Sigma \rightarrow I$ which connects directly the code space $\Sigma$ with the map $G$. For $n \in \mathbb{Z}$ let $e_{n}: \Sigma \rightarrow\left[-\omega^{-1}, 1\right]$ denote the evaluation map at time $n$ given by

$$
e_{n}(\mathbf{c})=-\sum_{k=n}^{\infty} c_{k}(-\omega)^{k-n}
$$

Then, clearly,

$$
e_{n}(\sigma(\mathbf{c}))=-\sum_{k=n}^{\infty} c_{k+1}(-\omega)^{k-n}=-\sum_{k^{\prime}=n+1}^{\infty} c_{k^{\prime}}(-\omega)^{k^{\prime}-(n+1)}=e_{n+1}(\mathbf{c}) .
$$

Writing e for the map of sequence spaces $\mathbf{e}: \Sigma \rightarrow\left[-\omega^{-1}, 1\right]^{\mathbb{Z}}$ given by $\mathbf{e}(\mathbf{c})_{n}=e_{n}(\mathbf{c})$, the relation (3.6) becomes

$$
\mathbf{e}(\sigma(\mathbf{c}))=\sigma(\mathbf{e}(\mathbf{c}))
$$

where we use $\sigma$ on the right hand side of this equation to denote the shift map on the space of sequences $\left[-\omega^{-1}, 1\right]^{\mathbb{Z}}$ where $\left[-\omega^{-1}, 1\right] \subseteq \mathbb{R}$.

The key property is that the image $\mathbf{e}(\Sigma)$ is precisely the set of full orbits of the map $G$ defined above. Indeed, away from the subinterval boundaries,

$$
e_{n}(\sigma(\mathbf{c}))=G\left(e_{n}(\mathbf{c})\right)
$$

\subsection{Partners and structures}

In this section we introduce an operation on codes in $\Sigma$ which we refer to as partnering. The purpose of partnering is to take into account the symmetries of the zeros of the initial conditions (2.19). These symmetries derive from the symmetry of the cosine function about $\pi$.

The partnering operation may seem somewhat strange and ad hoc, but in fact is related to the following identities satisfied by $\omega$.

From the defining equation of $\omega$ :

$$
\omega^{2}+a \omega-1=0,
$$


repeatedly multiplying through by $-\omega$ and adding itself readily gives the identities

$$
\begin{aligned}
& 1=a \omega+\omega^{2}, \\
& 1=(a+1) \omega-(a-1) \omega^{2}-\omega^{3}, \\
& 1=(a+1) \omega-a \omega^{2}+(a-1) \omega^{3}+\omega^{4}, \\
& 1=(a+1) \omega-a \omega^{2}+a \omega^{3}-(a-1) \omega^{4}-\omega^{5}, \\
& \ldots
\end{aligned}
$$

These identities are equivalent to the observation that 1 is a fixed point of the contractions

$$
\begin{aligned}
& \kappa_{2}(x)=a \omega+\omega^{2} x, \\
& \kappa_{3}(x)=(a+1) \omega-(a-1) \omega^{2}-\omega^{3} x, \\
& \kappa_{4}(x)=(a+1) \omega-a \omega^{2}+(a-1) \omega^{3}+\omega^{4} x, \\
& \kappa_{5}(x)=(a+1) \omega-a \omega^{2}+a \omega^{3}-(a-1) \omega^{4}-\omega^{5} x,
\end{aligned}
$$

We now define a substitution operation $\tilde{S}$ on biinfinite codes $\mathbf{c} \in \Sigma$ as follows. Firstly c is split into finite blocks beginning with a single 0 , i.e., into blocks of the form

$$
0 d_{0} d_{1} \ldots d_{k} 0^{r}
$$

where $k \geq 0, d_{j} \neq 0, j=0, \ldots, k, r \geq 0$. Further split the trailing run of 0 s so that this block is written

$$
\begin{aligned}
0 d_{0} d_{1} \ldots d_{k} 0(00)^{(r-1) / 2}, & r \text { odd } \\
0 d_{0} d_{1} \ldots d_{k}(00)^{r / 2}, & r \text { even } .
\end{aligned}
$$

On the resulting elementary blocks $00,0 d_{0} d_{1} \ldots d_{k}, 0 d_{0} d_{1} \ldots d_{k} 0$, the operation $\tilde{S}$ is then defined as follows.

$$
\begin{aligned}
\tilde{S}(0 d) & =0(a-d), \\
\tilde{S}\left(0 d_{0} d_{1}\right) & =0\left(a+1-d_{0}\right)\left(a-1-d_{1}\right), \quad k \geq 1, \\
\tilde{S}\left(0 d_{0} d_{1} \ldots d_{k-1} d_{k}\right) & =0\left(a+1-d_{0}\right)\left(a-d_{1}\right) \ldots\left(a-d_{k-1}\right)\left(a-1-d_{k}\right), \quad k \geq 2, \\
\tilde{S}\left(0 d_{0} d_{1} \ldots d_{k} 0\right) & =0\left(a+1-d_{0}\right)\left(a-d_{1}\right) \ldots\left(a-d_{k}\right)(a-1), \quad k \geq 1 .
\end{aligned}
$$

The operator $\tilde{S}$ preserves the number of digits and satisfies

$$
\tilde{S}(A B)=\tilde{S}(A) \tilde{S}(B)
$$

for any (finite or infinite) words $A, B$.

With the meaning that the $\alpha$-digit complement of $d \in\{0,1, \ldots, \alpha\}$ is $\bar{d}=\alpha-d$, so that $d+\bar{d}=\alpha$, the action of $\tilde{S}$ is to take digit complements according to the elementary block structures

$$
\begin{aligned}
& 0 a \\
& 0(a+1)(a-1) \\
& 0(a+1) a(a-1) \\
& 0(a+1) a a(a-1) \\
& \ldots
\end{aligned}
$$

For example, suppose $a=5$. Split

$$
\mathbf{c}=\ldots(011234)(05)(00)(03412330)(00) 051230 \ldots
$$

as indicated. Then

$$
\tilde{S}(\mathbf{c})=\ldots(054320)(00)(05)(03143224)(05) \tilde{S}(051230 \ldots)
$$


We have the following special cases.

(i) c begins with an infinite string $\ldots d_{-2} d_{-1} d_{0}$ containing no 0 . In this case we substitute this string to $\ldots\left(a-d_{-2}\right)\left(a-d_{-1}\right)\left(a-1-d_{0}\right)$.

(ii) c ends with an infinite string $d_{0} d_{1} d_{2} \ldots$ containing no 0 . Then in this case we substitute this string to $\left(a+1-d_{0}\right)\left(a-d_{1}\right)\left(a-d_{2}\right) \ldots$

(iii) $\mathbf{c}$ begins with the string $0^{\infty}$. Then we write $\mathbf{c}=(00)^{\infty} 0 d_{0} d_{1} \ldots, d_{0} \neq 0$ and substitute the string to $(0 a)^{\infty} \tilde{S}\left(0 d_{0} d_{1} \ldots\right)$.

(iv) $\mathbf{c}$ ends with the string $0^{\infty}$. We have an ambiguity in this case. When $\mathbf{c}=\ldots d_{-2} d_{-1} d_{0} 0^{\infty}$, with $d_{0} \neq 0$, we may write both $\mathbf{c}=\ldots d_{-2} d_{-1} d_{0}(00)^{\infty}$ and $\mathbf{c}=\ldots d_{-2} d_{-1} d_{0} 0(00)^{\infty}$. Then, respectively we have $\tilde{S}(\mathbf{c})=\tilde{S}\left(\ldots d_{-2} d_{-1} d_{0}\right)(0 a)^{\infty}, \tilde{S}(\mathbf{c})=\tilde{S}\left(\ldots d_{-2} d_{-1} d_{0} 0\right)(0 a)^{\infty}$. In the case $d_{-1}=0$, so that c $=\ldots d_{-2} 0 d_{0} 0^{\infty}$ then we could write both $\tilde{S}(\mathbf{c})=\tilde{S}\left(\ldots d_{-2}\right) 0\left(a-d_{0}\right)(0 a)^{\infty}$, and $\tilde{S}(\mathbf{c})=\tilde{S}\left(\ldots d_{-2}\right) 0\left(a-d_{0}\right)(a-1)(0 a)^{\infty}$. When $d_{-1} \neq 0$, then we could write both $\tilde{S}(\mathbf{c})=\ldots\left(a-1-d_{0}\right)(0 a)^{\infty}$, and $\tilde{S}(\mathbf{c})=\ldots\left(a-d_{0}\right)(a-1)(0 a)^{\infty}$. Cases (i) and (ii) are only possible when $a>1$.

For simplicity, we discard from $\Sigma$ these special cases and any code which may be obtained from them by using the partnering operation. By so doing, we discard a set that is nowhere dense in the standard topology on $\Sigma$. We shall use the notation $\tilde{\mathbf{c}}$ to denote the partner code $\tilde{S}(\mathbf{c})$.

We next define the sum map $\mathbf{S}: \Sigma \rightarrow\{1,-\omega-(a-1),-\omega-a\}^{\mathbb{Z}}$, the space of biinfinite sequences with terms taken from $\{1,-\omega-(a-1),-\omega-a\}$. $\mathbf{S}$ is defined in terms of the elementary block structures given above. Let $\mathbf{c}=\left(c_{k}\right)_{k \in \mathbb{Z}}$ be a code with partner $\tilde{\mathbf{c}}$. We divide $\mathbf{c}$ and $\tilde{\mathbf{c}}$ into blocks and define $\mathbf{S}$ on the elementary block structures $(3.26)-(3.30)$ as follows:

$$
\begin{aligned}
\mathbf{S}(0 a) & =1(-\omega-a) \\
\mathbf{S}(0(a+1)(a-1)) & =1(-\omega-a)(-\omega-(a-1)) \\
\mathbf{S}(0(a+1) a(a-1)) & =1(-\omega-a)(-\omega-(a-1))^{2} \\
\mathbf{S}(0(a+1) a a(a-1)) & =1(-\omega-a)(-\omega-(a-1))^{3}
\end{aligned}
$$

extending $\mathbf{S}$ to the whole of $\mathbf{c}$. By construction, we have that $\mathbf{S}(\mathbf{c})=\mathbf{S}(\tilde{\mathbf{c}})$ and $\mathbf{S}(\sigma(\mathbf{c}))=\sigma(\mathbf{S}(\mathbf{c}))$.

We have the following result which specifies precisely how $\mathbf{y}=\mathbf{e}(\mathbf{c})$ and $\tilde{\mathbf{y}}=\mathbf{e}(\tilde{\mathbf{c}})$ are related, and explains our terminology 'sum map'.

Proposition 1. Let $\mathbf{y}=\mathbf{e}(\mathbf{c})$ and $\tilde{\mathbf{y}}=\mathbf{e}(\tilde{\mathbf{c}})$. Then $\mathbf{y}+\tilde{\mathbf{y}}=\mathbf{S}(\mathbf{c})$, where, of course, the sum is to be calculated termwise.

To prove this proposition, let us consider a binfinite code $\mathbf{c}$ and its partner code $\tilde{\mathbf{c}}$, given by the above substitution rules. We write $y_{k}=e_{k}(\mathbf{c}), \tilde{y}_{k}=e_{k}(\tilde{\mathbf{c}})$ and set $S_{k}=y_{k}+\tilde{y}_{k}$. Let us write the codes in terms of the block structures explained above. Then we claim that if $n \in \mathbb{Z}$ starts a block (i.e., $c_{n}=\tilde{c}_{n}=0$, the first zero of a block), then $y_{n}+\tilde{y}_{n}=1$. Indeed, suppose the block starting at $n$ is of total length $j_{1} \geq 2$. Then we have $S_{n}=\kappa_{j_{1}}\left(S_{n+j_{1}}\right)$ so that $\left|S_{n}-1\right|=\left|\kappa_{j_{1}}\left(S_{n+j_{1}}\right)-\kappa_{j_{1}}(1)\right|=\omega^{j_{1}}\left|S_{n+j_{1}}-1\right|$. Since the next block, of total length $j_{2} \geq 2$ starts at $n+j_{1}$, we have, similarly, $\left|S_{n}-1\right|=\omega^{j_{1}+j_{2}}\left|S_{n+j_{1}+j_{2}}-1\right|$. Continuing in this way, and noting that the $S_{k}$ are bounded, gives in the limit $S_{n}=1$, as claimed.

We now consider $k \in \mathbb{Z}$ within a block. Consider an elementary block structure and let $n \in \mathbb{Z}$ correspond to the start of the following block. For the elementary block structure $0 a$ of length 2 , we have $S_{n-1}=$ $-a-\omega S_{n}=-a-\omega=\theta_{a}(1)$, since $S_{n}=1$. Thus $\mathbf{S}(0 a)=1(-\omega-a)$ corresponds to the sum $\mathbf{y}+\tilde{\mathbf{y}}$ on the block.

Similarly, for the elementary block structure $0(a+1)(a-1)$ of length 3 we have $S_{n-1}=-(a-1)-$ $\omega S_{n}=-\omega-(a-1)=\theta_{a-1}(1)$, and $S_{n-2}=-(a+1)-\omega S_{n-1}=\theta_{a+1}(-\omega-(a-1))=-\omega-a$, so that $\mathbf{S}(0(a+1)(a-1))=1(-\omega-a)(-\omega-(a-1))$ corresponds again to the sum $\mathbf{y}+\tilde{\mathbf{y}}$ on the block. (Here we have defined $\theta_{a+1}(x)=-\omega x-(a+1)$.) 
Finally, for an elementary block structure $0(a+1) a^{j}(a-1)$ of length $j+3$ for $j \geq 1$, we have $S_{n-1}=\theta_{a-1}(1)=$ $-\omega-(a-1), S_{n-1-j}=\theta_{a}(-\omega-(a-1))=-\omega-(a-1)$, since $-\omega-(a-1)$ is a fixed point of $\theta_{a}$. Finally, $S_{n-1-j-1}=\theta_{a+1}(-\omega-(a-1))=-\omega-a$, as before, so that $\mathbf{S}\left(0(a+1) a^{j}(a-1)\right)=1(-\omega-a)(-\omega-(a-1))^{j+1}$ corresponds again to the sum $\mathbf{y}+\tilde{\mathbf{y}}$ on the block.

This completes the proof of the proposition.

\section{Construction of the orchid}

In this section we outline our model of the orchid in the general $a$ case. Our basic tool is an embedding $\mathcal{E}$ of the space $\Sigma$ into a space $\mathcal{F}$ of function pairs $(u, t)$. The rigorous construction of this embedding in the case $a=1$ is given in [25], and, indeed, occupies a considerable part of that paper. We shall content ourselves here with a statement of the properties of the embedding map.

To simplify notation let us introduce the convention that binary and unary operations on pairs of functions are defined coordinatewise, so that, for example, for function-pairs $\left(u_{1}, t_{1}\right)$ and $\left(u_{2}, t_{2}\right)$, the product $\left(u_{1}, t_{1}\right)\left(u_{2}, t_{2}\right)$ denotes the function-pair $\left(u_{1} t_{1}, u_{2} t_{2}\right)$.

The philosophy given in Section 3.1 is that the zeros of the function-pair $(u, t)$ on $I$ determine the long-term dynamics of the pair $(u, t)$ and that the dynamics of the zeros themselves are governed by the map $G$. It is therefore not unexpected that the crucial property of the embedding $\mathcal{E}$ is that it takes a code c and maps it to a function pair that have zeros the value of the evaluation map $e_{0}(\mathbf{c})$. Indeed, our claim is that there is a map $\mathcal{E}: \Sigma \rightarrow \mathcal{F}$, relating the dynamics of $R$ to that of the shift map $\sigma$ on $\Sigma$, satisfying $\mathcal{E}(\mathbf{c})=(u, t)=$ $h_{0}(\mathbf{c})\left(u^{1}, t^{1}\right)$ where

$$
h_{0}(\mathbf{c})=\left\{\begin{array}{ll}
\left(y_{0}-x, y_{0}-x\right), & c_{-1}=0 \\
\left(1, y_{0}-x\right), & c_{-1} \neq 0
\end{array},\right.
$$

and $u^{1}, t^{1}$ are functions with $u^{1}(x)>0$ for $x \in I_{a}$ and $t^{1}(x)>0$ for $x \in I_{0} \cup \cdots \cup I_{a-1}$ and $y_{0}=e_{0}(\mathbf{c})$.

Let us define the following map $\kappa_{c}$ on sign-pairs. For $c \in\{0,1, \ldots, a\}$ define $\kappa_{c}:\{-1,+1\}^{2} \rightarrow\{-1,+1\}^{2}$ by

$$
\kappa_{c}\left(s^{u}, s^{t}\right)=\left(-s^{t},-(-1)^{c}\left(s^{t}\right)^{a} s^{u}\right) .
$$

It is easy to check that $\kappa_{c}$ is invertible with inverse $\kappa_{c}^{-1}\left(s^{u}, s^{t}\right)=\left(-(-1)^{c+a}\left(s^{u}\right)^{a} s^{t},-s^{u}\right)$.

The principal property of the map $\mathcal{E}$ is that it is a (semi)conjugacy to the renormalization operator $R$, up

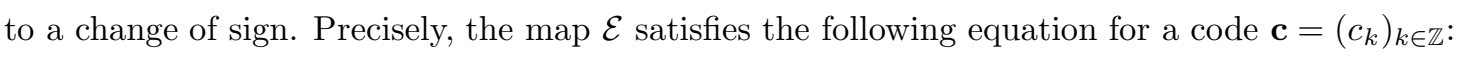

$$
R(\mathcal{E}(\mathbf{c}))=\kappa_{c_{0}}(+1,+1) \mathcal{E}(\sigma(\mathbf{c})) .
$$

The set $\mathcal{E}(\Sigma)$ does not correspond to the orchid, because for $\mathbf{c} \in \Sigma$, the function-pair $\mathcal{E}(\mathbf{c})$ does not have the correct zero structure. To rectify this problem we introduce a map $\beta: \Sigma \rightarrow \mathcal{F}$, defined by

$$
\beta(\mathbf{c})=\mathcal{E}(\mathbf{c}) \mathcal{E}(\tilde{\mathbf{c}})
$$

Corresponding to equation (4.3) there is a quasiconjugacy relation for the map $\beta$, but with a sign-pair evolution given by the map on sign-pairs $L_{b}$, given as follows. For $b \in\{0,1, \ldots, a+1\}$ define $L_{b}:\{-1,+1\}^{2} \rightarrow$ $\{-1,+1\}^{2}$ by

$$
L_{b}\left(s^{u}, s^{t}\right)=\left(s^{t},(-1)^{b}\left(s^{t}\right)^{a} s^{u}\right) .
$$

As before, it is easy to check that $L_{b}$ is invertible with inverse $L_{b}^{-1}\left(s^{u}, s^{t}\right)=\left((-1)^{b}\left(s^{u}\right)^{a} s^{t}, s^{u}\right)$. Then the quasiconjugacy relation for the map $\beta$ is

$$
R(\beta(\mathbf{c}))=L_{b_{0}}(+1,+1) \beta(\sigma(\mathbf{c})),
$$

where $b_{0}$ is the 0 th term of the sequence $\mathbf{b}=\mathbf{c}+\tilde{\mathbf{c}}$. Equation (4.6) shows the dynamics of $R$ can be modelled, up to a change of sign, by the shift map on $\Sigma$. We call the embedded set $\beta(\Sigma)$ the fundamental set of the orchid. Examples of these sets are shown in Figures 2, 3. 
We observe that $\beta(\mathbf{c})=\beta(\tilde{\mathbf{c}})$ for all $\mathbf{c} \in \Sigma$ and we may therefore define map on the quotient space $\Sigma^{\prime}$ obtained by identifying a code $\mathbf{c}$ with its partner code $\tilde{\mathbf{c}}$.

The orchid is itself made up of one or more copies of the fundamental set. We now explain the structure of the orchid set in terms of the fundamental set and the dynamics on the sign-pairs given by the map $L_{b}$.

For $\mathbf{c} \in \Sigma$ and $\left(s_{0}^{u}, s_{0}^{t}\right) \in\{-1,+1\}^{2}$ we define, for $n \in \mathbb{Z}$, the sign-pair $\left(s_{n}^{u}, s_{n}^{t}\right)=\left(s_{n}^{u}(\mathbf{c}), s_{n}^{t}(\mathbf{c})\right)$ by the condition

$$
L_{b_{n}}\left(s_{n}^{u}, s_{n}^{t}\right)=\left(s_{n+1}^{u}, s_{n+1}^{t}\right)
$$

for all $n \in \mathbb{Z}$, where, as before, $\mathbf{b}=\mathbf{c}+\tilde{\mathbf{c}}$.

In Figures 5, 6 are shown transition diagrams illustrating the dynamics of a sign-pair $\left(s^{u}, s^{t}\right) \in\{-1,+1\}^{2}$ under the maps $L_{b}$ for $\mathbf{c} \in \Sigma$. The sign-pairs in boxes correspond to the start of a block in the definition of the partner codes in Section 3.3. The map $L_{b}$ is applied to the sign-pair for each term in the block. The arrows represent transitions to other sign-pairs that may occur at the end of the block, the precise transition that occurs depends on the structure of the block. From these diagrams, it is evident that for $a$ odd, the four possible sign-pairs split into two components, both invariant under the transitions. One component is a single sign-pair and the other component consists of three sign-pairs. This structure explains the three-fold symmetry observed in the orchid for odd $a$. (See Figures 1, 3.) On the other hand, for $a$ even the sign-pairs split into three invariant components, one consisting of two sign-pairs and the other two consisting of a single sign-pair each.

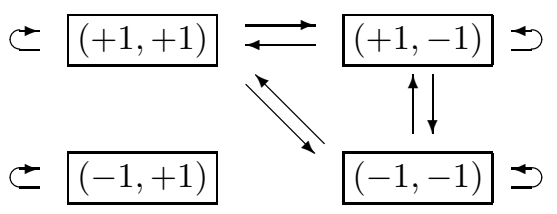

Figure 5: Sign transitions for $a$ odd.

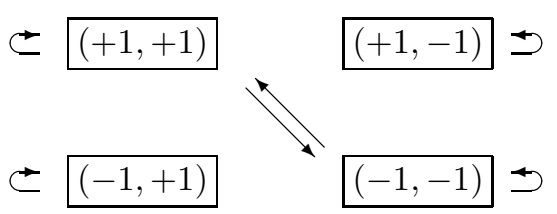

Figure 6: Sign transitions for $a$ even.

In order to determine which parts of these transition diagrams are relevant for the renormalization strange set for the generalized Harper equation, we must examine the zeros and poles of the initial condition (2.19). We observe first that we may separate out the numerator and the denominator of $t_{1}$ in (2.19). The denominator has zeros of order 2 at $x=1$ and $x=-\omega-(a-1)$, as may readily be checked. This give poles of order 2 for $t_{1}$. The function pair $\left(u_{2}, t_{2}\right)=R\left(u_{1}, t_{1}\right)$ consists of a function $u_{2}$ with a pole of order 2 at $-\omega-a$ and a function $t_{2}$ with poles of order 2 at 1 and $-\omega-a$. This configuration of poles is fixed under $R$, leading to the function-pair $\left(u_{n}, t_{n}\right)$ converging to a fixed-point pair $(u, t)$ with the same configuration of poles.

The numerator of $t_{1}$ gives the orchid-like structure, and we now consider the zeros of $t_{1}$. Let $\alpha>1$, so that we may write $\alpha=1 / \cos (2 \pi r)$, for $0<r<1 / 4$. The roots of $t_{1}(x)$ in $I$ are $x_{ \pm}=-(1 / 2 \mp r-\omega / 2) / \omega$ and $\left(u_{1}(x), t_{1}(x)\right)=\left(1,\left(x_{+}-x\right)\left(x_{-}-x\right)\right)\left(u^{1}(x), t^{1}(x)\right)$ where $u^{1}, t^{1}$ are positive on $I_{a}$ and $I_{0} \cup \cdots \cup I_{a-1}$ respectively. Thus we have the sign-pair $\left(s^{u}, s^{t}\right)=(+1,+1)$. We note that $x_{+}+x_{-}=-\omega-(a-1)$. This means that the initial condition is not at the start of a block, and one must iterate the recurrence in order to see which part of the transition diagram the initial condition corresponds to. The cases of odd and even $a$ are different. For $a$ odd, we obtain the full three-fold symmetrical orchid, whilst for $a$ even, the orbit is restricted to a single copy of the fundamental set. 
To understand the reason for this, we observe that, since $x_{+}+x_{-}=-\omega-(a-1)$, the initial condition $\left(u_{1}, t_{1}\right)$ corresponds to one of the final $j+1$ positions in an elementary block structure of the form $0(a+1) a^{j}(a+1)$ of length $j+3$ for $j \geq 0$. Let us now consider the action of the map $L_{b}$ on the sign-pair $(+1,+1)$ for $b=a$, for $k$ iterations, $0 \leq k \leq j$ and $b=a+1$ for a final, single iteration to bring the function pair to the start of the following block.

For odd $a>1$, the sign-pair $(+1,+1)$ cycles through the sequence $(+1,+1),(+1,-1),(-1,+1)$ for $k$ iterations of the map $L_{a}$. The final iteration with the map $L_{a+1}$ maps this sequence to $(+1,+1),(-1,-1),(+1,-1)$, one of which will be the sign-pair at the start of the next block. Referring to Figure 5, we see that this corresponds to the invariant component with three sign-pairs, and hence we have a threefold symmetry in this case, as for the golden-mean orchid.

However, for even $a>1$, the sign-pair $(+1,+1)$ is unaffected by the maps $L_{a}$, but $L_{a+1}(+1,+1)=(+1,-1)$, which is the signpair at the start of the next block. Referring to the transition diagram Figure 6 , we see that we obtain a single copy of the fundamental set.

Although the initial condition for the generalized Harper equation gives a single copy of the fundamental set for even $a$, by appropriate choice of the initial sign-pair one can obtain a renormalization strange set with two-fold symmetry, as illustrated in Figure 7.

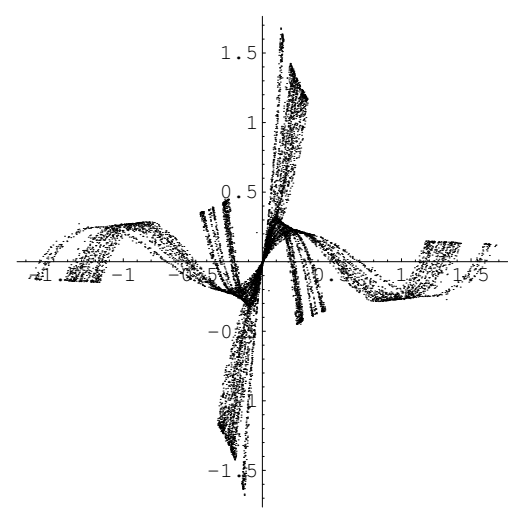

Figure 7: An invariant set with twofold symmetry that may occur in the case $a=2$.

\section{Construction of the map $\mathcal{E}$}

The construction of the map $\mathcal{E}$ follows standard methods for the construction of conjugacies, in that the map is obtained as a limit of backwards and forwards iteration.

Let $\mathbf{c} \in \Sigma$ and let $h_{-k}(\mathbf{c})=h_{0}\left(\sigma^{-k}(\mathbf{c})\right)$, where $h_{0}$ is given by equation (4.1). We also choose a sign-pair $\left(s_{-k}^{u}, s_{-k}^{t}\right)=\kappa_{c_{-k}}^{-1} \circ \cdots \circ \kappa_{c_{-1}}^{-1}(+1,+1)$. We define the map

$$
\mathcal{E}(\mathbf{c})=\lim _{k \rightarrow \infty} R^{k} P\left(s_{-k}^{u}, s_{-k}^{t}\right) h_{-k}(\mathbf{c}),
$$

where $P$ is the projection operator defined below. We remark that the proof of convergence (given in [25] for the golden-mean case) is rather lengthy.

We now give details of the projection $P$. The renormalization transformation $R$ is not a contraction on the full space of function pairs; indeed its linearized version has two non-contracting directions. Thus, in order to obtain convergence for smooth functions will shall need to project down to the stable manifold. 
Defining

$$
\begin{aligned}
\Delta_{n}(U, T) & =\int_{-\omega^{-1}}^{1-\omega^{-1}} U^{(n)}(x) d x+\int_{1-\omega^{-1}}^{1} T^{(n)}(x) d x, \\
\mathbf{v}_{0} & =\frac{1}{\omega+\omega^{-1}}(\omega, 1), \\
\mathbf{v}_{1} & =\frac{1}{\omega+\omega^{-1}}(\omega x-\omega-(a-1) / 2, x+\omega+(a-1) / 2),
\end{aligned}
$$

the following properties may be verified:

1. $\Delta_{n}\left(R_{a}(U, T)\right)=(-1)^{n} \omega^{n-1} \Delta_{n}(U, T)$ for $n=0,1$;

2. $\Delta_{0}\left(\mathbf{v}_{0}\right)=1, \quad \Delta_{0}\left(\mathbf{v}_{1}\right)=0, \quad \Delta_{1}\left(\mathbf{v}_{0}\right)=0, \quad \Delta_{1}\left(\mathbf{v}_{1}\right)=1$.

Let us introduce the following projection operator $P$, defined by

$$
P(u, t)=(u, t) \exp \left(-\Delta_{0}(\log |(u, t)|) \mathbf{v}_{0}-\Delta_{1}(\log |(u, t)|) \mathbf{v}_{1}\right) .
$$

Then, by a straightforward calculation, one may show that $P$ satisfies the following properties:

1. $P^{2}=P$;

2. $P R=R P$;

3. $P\left(\left(u_{1}, t_{1}\right)\left(u_{2}, t_{2}\right)\right)=P\left(u_{1}, t_{1}\right) P\left(u_{2}, t_{2}\right)$.

The initial condition $\left(u_{1}, t_{1}\right)$ satisfies $P\left(u_{1}, t_{1}\right)=\left(u_{1}, t_{1}\right)$, as may be readily verified by direct calculation, using the integral identity

$$
\int_{A}^{A+2 \pi / B} \log |1+B \cos (C x+D)| d x=\frac{2 \pi}{C} \log (|B| / 2)
$$

for $A, B, C, D \in \mathbb{R}$ with $|B| \geq 1, C>0$, to show $\Delta_{0}\left(\log \left|\left(u_{1}, t_{1}\right)\right|\right)=0$, and taking appropriate limits to show $\Delta_{1}\left(\log \left|\left(u_{1}, t_{1}\right)\right|\right)=0$. Thus $\left(u_{1}, t_{1}\right)$ lies on the stable manifold of the renormalization strange set.

We remark that the operator $R P$ may also be used to construct the orchid numerically, although care must be taken to preserve the symmetry of the zero set. Such a method was used to obtain the pictures shown in Figures $1-3$.

\section{Discussion}

In this paper we have considered the generalized Harper equation for quadratic irrationals $\omega=\left(\sqrt{a^{2}+4}-\right.$ a) $/ 2$ for general $a \in \mathbb{N}$. We have presented new orchid-like renormalization strange sets for each value of $a$ and have given model structure for these sets in terms of biinfinite codes for the fundamental set. The orchid itself is constituted of one or more copies of the fundamental set, with the precise number of copies emerging from an analysis of the dynamics on the sign-pairs, as given by the transition diagrams (Figure 5, $6)$.

The structure of the orchid depends in an essential way on the symmetries of the generalized Harper equation (1.1), and in particular on those of the cosine function. It is these symmetries that lead to the partnering operation on codes and the relation on the zeros of the function pairs $\beta(\mathbf{c})$. If one destroys the symmetries, then, likewise, the orchid is destroyed and is replaced by a different strange set. Indeed, when calculating the orchid numerically, care must be taken with round-off error in the zeros of the function pairs. Since the chaotic map $G$ governs the dynamics of these zeros, round off error grows quickly leading to non-convergence to the orchid. Thus the universality class for the orchid is restricted to those quantum models displaying the same symmetries as the generalized Harper equation model (1.1). Moreover, the existence of non-stable 
eigendirections for the operator $R_{a}$ implies further conditions on a function pair $(u, t)$ to converge to the orchid. These conditions are satisfied for the initial conditions derived from the generalized Harper equation model, but it is clearly a moot point to what extent one should refer to the orchid as a renormalization strange 'attractor.'

The ideas and techniques in this paper are likely to find application in other problems in the field. As first pointed out by Bondeson et al [2], there is an equivalence between the transition to the localized state in quasiperiodic Schrödinger equations and the onset of a strange nonchaotic attractor in quasiperiodically forced nonlinear systems. See also [17]. Kuznetsov et al [21] have given a renormalization analysis of the onset of a strange nonchaotic attractor. We anticipate that our work in this paper will shed considerable light on this related problem, and in particular we expect that the numerical results in [21] can be generalized and put on a rigorous foundation. Rigorous renormalization analyses of correlations in strange nonchaotic attractors [7] and in quasiperiodically forced two-level system [8] have recently been completed [23, 24].

The case of more general irrational $\omega$ is an important next step in the work in this area. It is clear that the work presented here may be readily generalized to all quadratic irrational $\omega$, i.e., to all those $\omega$ having periodic tails in their continued-fraction expansions. A harder problem is the extension of the theory to more general irrational frequencies. It is likely that for general $\omega$ the functions $t_{n}$ (and thus the fluctuations $\eta_{i}$ ) move chaotically between the orchids presented here for different $a$, with the renormalization of $\omega$ governed by the Gauss map $\gamma(x)=1 / x-[1 / x]$. This is analogous to other quasiperiodic renormalizations such as critical circle and area-preserving maps. The analysis for general $\omega$ presents a considerable challenge for future work in this field.

Let us conclude with a few remarks concerning the potential physical application of the theory, for, in common with many other areas of mathematical physics, it is questionable to what extent the phenomena described here arise in an actual physical system.

Firstly, the generalized Harper equation is itself a discrete approximate model of an idealized chain of atoms in a sinusoidal applied potential, in which only nearest and next-nearest-neighbour interactions are considered. Perturbation of the model destroys the symmetries of the cosine function, and thus the global structure of the strange set. Secondly, the structures presented here correspond to frequencies taken from a special class of quadratic irrationals, which themselves constitute a measure-zero subset of all frequencies. Moreover, they govern asymptotic correlations in the fluctuations in an exponentially decaying wave-function, and only between certain atom sites related by the rational convergents to the frequency.

Of course, these same criticisms apply in many other branches of physics as well, and indeed the whole of science abounds with non-generic models and theories, which despite their unreasonableness, are remarkably successful. Moreover, in many quasiperiodic systems quadratic irrationals (and indeed the golden mean, in particular) seem to dominate the dynamical behaviour. Furthermore, experimentalists have been quite successful in testing other renormalization theories that are frequency dependent (see, for example, [26]), and indeed it may be possible to conduct similar experiments in this case. Moreover, the results in this paper are likely to carry over to more general frequency.

More problematic may be the difficulty of accurately measuring the fluctuations in exponentially decaying wave-functions, which themselves can only be inferred by probabilities obtained in repeated experiments. However, we expect the strong-coupling results of our analysis here to be mirrored in the fat critical phase of the model (see [15]) in which the fluctuation are in the wave-function itself. In addition, of course, it may be that a deeper analysis of the localized phase could identify macroscopic properties of materials that are influenced by the correlations considered here, but at present this is pure speculation.

\section{References}

[1] S. Aubry and G. André, Analyticity breaking and Anderson localization in incommensurate lattices, Ann. Israel Phys. Soc. 3 (1980), 133-164. 
[2] A. Bondeson, E. Ott, and T. M. Antonsen, Quasiperiodically forced damped pendula and Schrödinger equations with quasiperiodic potentials: implications of their equivalence, Phys. Rev. B 55 (1985), 21032106.

[3] J. R. Chapman and A. H. Osbaldestin, Self-similar correlations in a barrier billiard, Physica D 180 (2003), 71-91.

[4] F. H. Claro and G. H. Wannier, Magnetic subband structure of electrons in a hexagonal lattice, Phys. Rev. B 19 (1979), 6068-6074.

[5] J. Dalton and B. D. Mestel, Renormalization for the Harper equation for quadratic irrationals, J. Math. Phys. 44 (2003), 4776-4783.

[6] D. Domínguez, C. Wiecko, and J. V. José, Wave-function and resistance scaling for quadratic irrationals in Harper's equation, Phys. Rev. B 45 (1992), 13919-13929.

[7] U. Feudel, A. Pikovsky, and A. Politi, Renormalization of correlations and spectra of a strange nonchaotic attractor, J. Phys. A 29 (1996), 5297-5311.

[8] U. Feudel, A.S. Pikovsky, and M. A. Zaks, Correlation properties of a quasiperiodically forced two-level system, Phys. Rev. E 51 (1995), 1762-1769.

[9] J. H. Han, D. J. Thouless, H. Hiramoto, and M. Kohmoto, Critical and bicritical properties of Harper's equation with next-nearest-neighbor coupling, Phys. Rev. B 50 (1994), 11365-11380.

[10] P. G. Harper, Single band motion of conduction electrons in a uniform magnetic field, Proc. Phys. Soc. London A 68 (1955), 874-892.

[11] Y. Hatsugai and K. Kohmoto, Energy spectrum and the quantum Hall effect on the square lattice with next-nearest-neighbor hopping, Phys. Rev. B 42 (1990), 8282-8294.

[12] J. A. Ketoja, Universal criterion for the breakup of invariant tori in dissipative systems, Phys. Rev. Lett. 69 (1992), 2180-2183.

[13] J. A. Ketoja and I. I. Satija, Renormalization approach to quasiperiodic tight binding models, Phys. Lett. A 194 (1994), 63-70.

[14] J. A. Ketoja and I. I. Satija, Renormalization approach to quasiperiodic quantum spin chains, Physica A 219 (1995), 212-233.

[15] J. A. Ketoja and I. I. Satija, Self-similarity and localization, Phys. Rev. Lett. 75 (1995), 2762-2765.

[16] J. A. Ketoja and I. I. Satija, The re-entrant phase diagram of the generalized Harper equation, J. Phys. C 9 (1997), 1132.

[17] J. A. Ketoja and I. I. Satija, Harper equation, the dissipative standard map and strange nonchaotic attractors: Relationship between an eigenvalue problem and iterated maps, Physica D 109 (1997), 7080.

[18] J. A. Ketoja, I. I. Satija, and J. C. Chaves, Decimation studies of Bloch electrons in a magnetic field: Higher-order limit cycles underlying the phase diagram, Phys. Rev. B 52 (1995), 3026-3029.

[19] M. Kohmoto, Matel-insulator transition and scaling for incommensurate systems, Phys. Rev. Lett. 51 (1983), 1198-1201.

[20] M. Kohmoto, L. P. Kadanoff, and C. Tang, Localization problem in one-dimension: mapping and escape, Phys. Rev. Lett. 50 (1983), 1870-1872. 
[21] S. P. Kuznetsov, A. S. Pikovsky, and U. Feudel, Birth of a strange nonchaotic attractor: A renormalization group analysis, Phys. Rev. E 51 (1995), R1629-R1632.

[22] B. D. Mestel, A. H. Osbaldestin, and B. Winn, Golden mean renormalisation for the Harper equation: the strong coupling fixed point, J. Math. Phys. 41 (2000), 8304-8330.

[23] B. D. Mestel and A. H. Osbaldestin, Periodic orbits of renormalisation for the correlations of strange nonchaotic attractors, Math. Phys. EJ 6 (2000), no. 5, 27 pp.

[24] B. D. Mestel and A. H. Osbaldestin, Renormalization analysis of correlation properties in a quasiperiodically forced two-level system, J. Math. Phys. 43 (2002), 3458-3483.

[25] B. D. Mestel and A. H. Osbaldestin, Golden mean renormalization for a generalized Harper equation: the Ketoja-Satija orchid, preprint (2003).

http://www.maths.stir.ac.uk/ bdm/research/publications/orchid.pdf

[26] J. Stavans, F. Heslot, and A. Libchaber, Fixed winding number and the quasiperiodic route to chaos in a convective fluid, Phys. Rev. Lett. 55 (1985), 596-599 and 1239.

[27] D. J. Thouless, Bandwidths for a quasiperiodic tight-binding model, Phys. Rev. B 28 (1983), 4272-4276. 